\title{
Transport des protéines plasmatiques à travers la paroi artérielle et athérogenèse
}

L'accumulation de lipoprotéines dans l'intima des grosses artères est un des facteurs déclenchants de l'athérosclérose; elle est la résultante de l'influx en provenance du plasma circulant (augmenté en cas d'hypertension artérielle, d'hypercholestérolémie, de lésion de l'endothélium) et de l'efflux vers l'adventice à travers la média musculaire (diminué en cas d'épaississement de la média provoqué par une hypertension artérielle chronique).

Joël Belmin Alain Tedgui

\section{ADRESSE}

J. Belmin : Chef de clinique. Hôpital Charles Foix, 7, avenue de la République, 94400 Ivry, France. A. Tedgui : Chargé de recherche à l'Inserm. Inserm U. 14l, hôpital Lariboisière, 41, boulevard de la Chapelle, 75010 Paris, e pathologiste Rudolf Virchow (1821-1902)[1] a donné naissance aux deux grands courants de pensée l'étiologie de l'athérosclérose. Selon lui, en effet, les lipides plasmatiques sont absorbés directement à travers l'endothélium par l'intima et, lorsque les conditions le permettent, certains d'entre eux, en particulier les esters de cholestérol, s'y déposent. C'est l'hypothèse de l'infiltration lipidique comme facteur initiateur de la plaque d'athérome. Virchow pensait également qu'à l'origine de cette infiltration lipidique, il y avait traumatisme endothélial provoqué par des facteurs mécaniques. C'est sur cette deuxième hypothèse que reposent la théorie de la « réponse à l'effraction endothéliale » de Russel Ross [2] et, de façon plus générale, les théories dites «cellulaires » de l'athérogenèse.

En 1954, Irving Page [3] reprend et développe l'idée de l'infiltration lipi- . dique: «Le concept de filtration repose sur la vision que l'athérogenèse est le résultat d'une réaction tissulaire à des substances filtrées à partir du plasma, telles que les lipo- protéines, sous l'effet de la pression artérielle latérale, et déposées dans l'intima comme agents étrangers. La plupart des substances filtrées passent sans difficulté à travers la paroi pour être récupérées par les capillaires adventiciels ou la lymphe. Mais il se peut que certaines d'entre elles puissent s'immobiliser, soit parce que les propriétés de filtre du vaisseau sont altérées, soit parce que la taille, la forme et la charge des lipoprotéines font qu'elles adhèrent au sous-endothélium. Des variations dans l'arrangement, la quantité et la nature chimique de la substance fondamentale sous-endothéliale peuvent être à l'origine d'une modification focale de la fonction de filtre de la paroi. La réaction qui s'ensuit dépend de la nature du lipide déposé et de la réponse tissulaire. » Irving Page précise ensuite que « les altérations de la capacité de filtration de la paroi vasculaire peuvent être la conséquence du vieillissement, de l'hypertension ou de désordres métaboliques. »

Cet auteur fut le premier à mettre aussi clairement l'accent sur l'importance, dans l'athérogenèse, du transport transpariétal des substances 


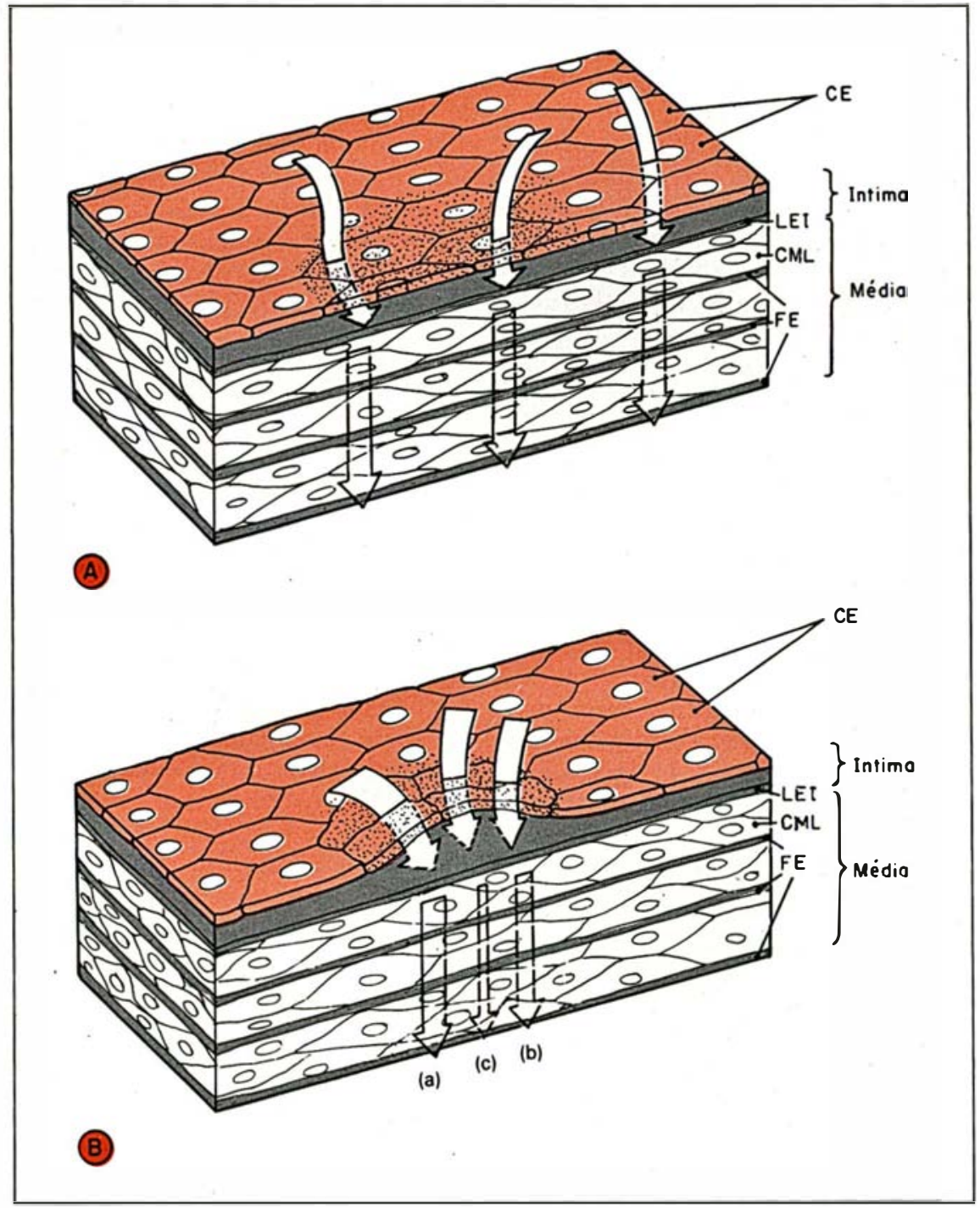

plasmatiques. Or, la preuve que la média des grosses artères offre en effet une résistance importante au transfert de masse vers l'adventice ne fut apportée que récemment [4]. Smith et Staples [5] rapportent des taux de $\mathrm{LDL}^{*}$ deux fois plus élevés dans le liquide interstitiel de l'intima d'artères humaines que dans le plasma, laissant penser que la limitante élastique interne et la média tout entière empêchent les lipoprotéines d'être évacuées vers les lymphatiques adventiciels.

Il est intéressant de remarquer à cet égard que seules les grosses artères à média épaisse sont touchées par l'athérome, tandis que les artères de

- LDL: low density lipoproteins, lipoprotéines de faible densité.

$\mathrm{m} / \mathrm{s} n^{\circ} 1$ vol. 5 , janvier 89 petit calibre, les artérioles et les veines sont épargnées, bien que l'environnement plasmatique (en particulier, en cholestérol) soit le même pour tous ces vaisseaux. Dès 1952, d'ailleurs, Wilens et McCluskey [6] suggéraient que « les différences d'incidences des dépôts lipidiques observées entre les artères et les veines sont liées à des différences de perméabilité. » Et, en effet, si on limite expérimentalement la sortie des macromolécules de la paroi de la veine, il est possible d'y engendrer des lésions athéromateuses [7].

L'accumulation lipidique sousendothéliale, observée au stade le plus précoce de l'athérosclérose, est probablement le résultat d'un déséquilibre entre l'entrée et la sortie des LDL plasmatiques, l'entrée étant
Figure 1. Schéma illustrant la relation entre les perméabilités de l'endothélium et de la média et l'apparition des lésions d'athérome. Les cellules endothéliales (CE) sont de forme losangique et leur grand axe est orienté dans le sens de l'écoulement. A. Dans la situation normale, les flux d'entrée des macromolécules plasmatiques à travers l'endothélium (flèches grises) et de sortie à travers la média (flèches rouges) sont d'importance égale. B. Dans les situations pathologiques, l'accumulation de substances plasmatiques dans l'espace situé entre l'endothélium et la limitante élastique interne (LEI) peut résulter, soit d'une augmentation du flux d'entrée à travers l'endothélium, le flux de sortie étant inchangé (a), soit d'une diminution du flux de sortie, le flux d'entrée restant normal (b), soit d'une augmentation de l'entrée et d'une diminution de la sortie (c). CML : cellule musculaire lisse, FE : fibre élastique. contrôlée par l'endothélium et la sortie dépendant de la perméabilité de la média (figure 1). Afin de comprendre l'initiation de la plaque d'athérome et d'élucider les mécanismes responsables des dépôts lipidiques dans l'intima, de nombreux travaux ont été consacrés à l'étude du transport des protéines plasmatiques, et des LDL en particulier, à travers la paroi des vaisseaux. Cet article propose une revue des données actuelles concernant cet aspect méconnu de la biologie de la paroi artérielle.

\section{Méthodes d'étude}

En 1922, Petroff [8] observe que l'injection de bleu d'Evans, un colorant qui se lie fortement à l'albumine, colore préférentiellement certaines 


\section{RÉFÉRENCES}

1. Virchow R. Gesammelte Abhanndlungen zür wissenschaftlichen Medizin. In : Phlogose und Thrombose im Gefassystem. Berlin : Max Hirsch, 1862 : 458-521. Traduit en appendice dans Pickering G. Pathogenesis of myocardial and cerebral infarction : nodular arteriosclerosis. Br Med J 1964 ; 1 : 517-29.

2. Ross R.' The pathogenesis of atherosclerosis. An update. N Engl J Med 1986 ; 314 : 488500

3. Page IH. Atherosclerosis. An introduction. Circulation $1954 ; 10$ : 1-27.

4. Caro CG, Lever MJ, Laver-Rudich Z, et al. $\mathrm{Net}$ albumin transport across the wall of the rabbit common carotid artery perfused in situ. Atherosclerosis $1980 ; 37: 497-511$

5. Smith EB, Staples EM. Distribution of plasma proteins across the human aortic wall. Barrier function of endothelium and internal elastic lamina. Atherosclerosis 1980 ; 37 : $579-90$

6. Wilens SL, McCluskey RT. The comparative filtration properties of excised arteries and veins. Am J Med Sci 1952 ; 224 : 540-7.

7. Curmi PA, Tedgui A. Evidence of vascular macromolecular clearance as an atherogenic factor in the rabbit inferior vena cava and abdom inal aorta. J Physiol (Lond) 1987 ; 388 : $23 \mathrm{P}$.

8. Petroff IR. U'ber die Vitalfarbung der Gefasswandungen. Beitr.Path Anat 1922 ; 71 : 115-31.

9. Bell FP, Adamson IL, Schwartz CJ. Aortic endothelial permeability to albumin: focal and regional patterns of uptake and transmural distribution of ${ }^{125} \mathrm{l}$-albumin in the young pig. Exp Mol Pathol 1974 ; 20 : 57-68.

10. Friedman M, Byers SO. Endothelial permeability in atherosclerosis. Arch Pathol $1963 ; 63$ : 99-105.

11. Gerrity RG, Naito HK, Richardson M, Schwartz CJ. Dietary induced atherogenesis in swine: morphology of the intima in swine: morphology of the intima in prelesion stages. Am J Pathol 1979 ; 95 : 775-86.

12. Hoff HF, GerrityRG, Niato HK, Dusek DM. Quantification of apolipoprotein

B in aortas of hypercholesterolemic swine. Lab Invest 1983 ; 48 : 492-504.

13. Armstrong ML, Megan MB, Warner ED. The relation of hypercholesterolemic fatty streaks to intimal permeability changes shown by Evans blue. Atherosclerosis 1978; $31: 443-52$

14. Bratzler RL, Chilsom GM, Colton CK, Smith KA, Lees RS. The distribution of labeled low density lipoproteins across the rabbit thoracic aorta in vivo. Atherosclerosis 1977 ; 28 : 289-307.

15. Bratzler RL, Chilsom GM, Colton CK, Smith KA, Zilversmit DB, Lees RS. The distribution of labeled albumin across the rabbit thoracic aorta in vivo. Circ Res 1977 ; 40 : 182 90.

16. Tedgui A, Lever MJ. The interaction of convection and diffusion in the transport of

131l-albumin within the media of the rabbit thoracic aorta. Circ Res $1985 ; 57$ : 856-63.

17. Tedgui A, Chiron B, Curmi P, Juan L. Effect of nicardipine and verapamil on in vivo albumin transport in rabbit thoracic zones de la face luminale de l'aorte. D'autres travaux ont confirmé ultérieurement ces résultats chez différentes espèces animales, et ont montré que ces zones colorées correspondent à des territoires dont la perméabilité à l'albumine est augmentée [9]. De plus, la distribution globale de ces zones colorées semble s'apparenter à celle des plaques d'athérome induites chez l'animal par un régime enrichi en cholestérol [10-12], même si certaines études rapportent une dissociation entre l'apparition de la strie lipidique et l'augmentation de la perméabilité au bleu d'Evans [13]. Cette approche méthodologique comportait toutefois de nombreuses limites; elle ne renseigne pas sur l'origine de l'augmentation de la captation d'albumine par la paroi : augmentation de la perméabilité endothéliale ou réduction de la perméabilité de la média, ou les deux à la fois (voir figure 1). De plus, ces résultats ne sont guère quantitatifs. C'est pourquoi, à la suite de ces travaux, plusieurs méthodes ont été développées pour étudier de façon plus fine les déterminants de ce transport. Leur principe général consiste à suivre la pénétration dans la paroi artérielle d'un traceur protéique.

Traceur protéique. Les traceurs protéiques les plus utilisés sont l'albumine ou les LDL radio-actives (marquées à l'iode) et la peroxydase du raifort. Leur présence dans la paroi est révélée par comptage de la radioactivité pour l'albumine et pour les LDL, et par réaction enzymatique pour la peroxydase. Leurs tailles ( $4 \mathrm{~nm}$ de diamètre pour la peroxydase, $7 \mathrm{~nm}$ pour l'albumine et $20 \mathrm{~nm}$ pour les LDL) doivent être prises en compte, car le transport est d'autant plus important que la molécule est petite. Enfin, les concentrations de LDL dans la paroi sont déterminées, non seulement par leur transport passif à travers la paroi, mais aussi par la liaison et la dégradation cellulaires in situ, liées à la présence de récepteurs spécifiques aux LDL, contrairement aux deux autres traceurs qui ne sont pas catabolisés in situ et peuvent être considérés comme quasiment inertes.

Les traceurs radio-actifs ont pour avantages une utilisation assez sim- ple et une grande précision, sous réserve de quelques précautions telles que le contrôle de la stabilité de la liaison entre protéine et molécule radio-active. L'albumine marquée est le traceur de choix pour l'étude des mécanismes passifs du transport macromoléculaire, en raison de la similarité de sa pénétration avec celle des $\operatorname{LDL}[14,15]$.

Concentrations pariétales en traceurs. La distribution transmurale des concentrations en protéines plasmatiques n'est pas homogène à travers la paroi, si bien qu'une mesure globale de la concentration est peu informative et peut même conduire à des interprétations fausses. Des techniques de coupes sériées en cryocongélation ont été développées. Sur des segments d'aorte mis à plat, des sections dont l'épaisseur peut atteindre $10 \mu \mathrm{m}$ sont effectuées parallèlement à la surface endothéliale. Les concentrations en traceur protéique sont mesurées dans chacune des sections (concentrations tissulaires) et rapportées à la concentration plasmatique (concentrations tissulaires relatives). On obtient ainsi une distribution transmurale des concentrations en traceur dans la paroi depuis sa face luminale jusqu'à sa face adventicielle [4, 15-17] (figure 2).

Un changement dans l'aspect macroscopique des coupes permet de repérer la transition entre média et adventice. La principale limite de cette technique est l'impossibilité d'obtenir des concentrations dans l'intima. En effet, cette couche est particulièrement fine ( 5 à $7 \mu \mathrm{m}$ chez le lapin ou le porc) et la première coupe effectuée englobe à la fois l'intima et la zone juxta-intimale de la média. De plus, compte tenu de la méthode utilisée, l'épaisseur de cette première coupe peut être inférieure à l'épaisseur fixée, ce qui peut entrâ̂ner une sous-estimation inconnue de la concentration tissularre dans cette coupe. Toutefois, dans l'aorte humaine, dont l'intima est plus épaisse, ces concentrations en LDL et en albumine ont pu être mesurées et fournissent de précieux renseignements $[5,18]$.

Modèles expérimentaux. Le transport d'un traceur protéique dans la paroi artérielle peut être étudié soit 


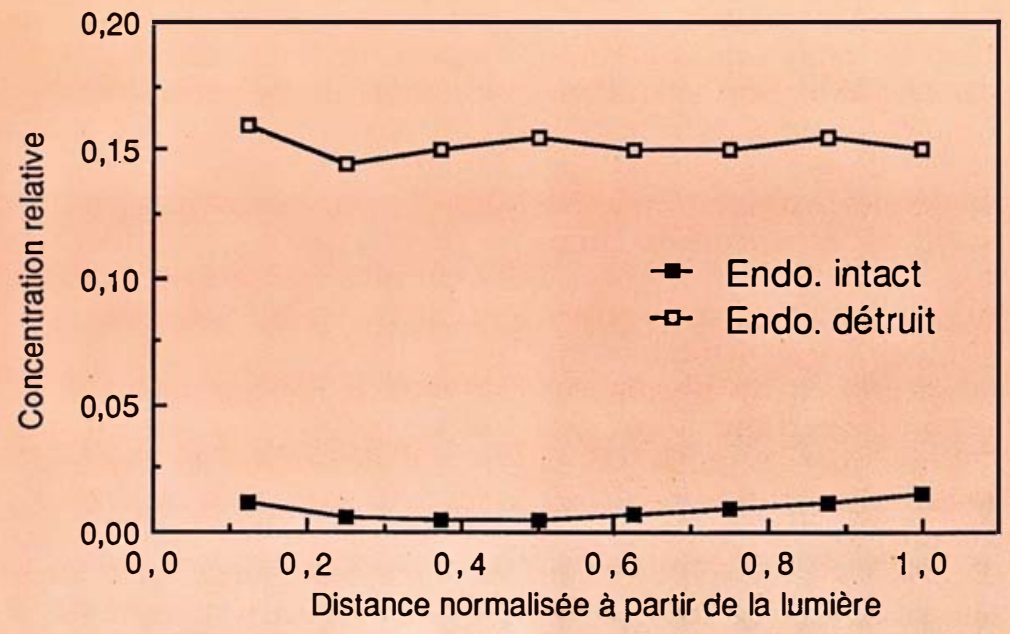

Figure 2. Distribution transmurale de la concentration relative d'albumine à travers la média de l'aorte de lapin avec (Endo. intact) et sans endothélium (Endo détruit). La distance à partir de la surface luminale est normalisée par l'épaisseur de la média ; 1,0 en abscisse représente la limite entre la média et l'adventice.

chez l'animal in vivo, soit sur des artères isolées in vitro. Les artères les plus étudiées sont l'aorte et la carotide primitive. Pour l'étude in vivo, on injecte à l'animal le traceur protéique par voie veineuse. La durée de l'expérience doit être fixée en fonction de deux impératifs : suffisamment longue pour que les concentrations dans la paroi soient à l'équilibre, mais suffisamment courte pour que les concentrations plasmatiques de traceur restent stables. Après sacrifice de l'animal, l'artère doit être prélevée très rapidement et être aussitôt mise à plat et congelée [14, 15].

L'étude du transport sur des artères excisées in vitro est très précieuse surtout en ce qui concerne l'étude des mécanismes de ce transport. Elle permet de contrôler les facteurs influençant le transport, indépendamment les uns des autres, tels que la pression intra-artérielle, la dénudation endothéliale, les agents vasopresseurs ou pharmacologiques ou même d'autres facteurs hémodynamiques tels que la vélocité sanguine ou la composante oscillatoire de la pression artérielle. De plus, il est possible d'appliquer le traceur soit à l'intérieur de l'artère, soit à l'extérieur de l'artère (ce qui n'est pas réalisable in vivo), soit sur les deux faces à la fois afin de mesurer le volume de distribution de l'espèce considérée $[16,19]$. Toutefois, l'obtention de ces artères excisées in vitro est très difficile en pratique en raison de la grande fragilité de l'endothélium. Pour ne pas léser cette structure, il est indispensable, pendant le prélèvement, de maintenir dans l'artère une pression voisine de la pression artérielle moyenne, ce qui impose une ligature de toutes les collatérales et une perfusion préalable de l'artère. De plus, pour ne pas être déformée, l'artère est fixée sur un support rigide avant d'être prélevée et placée dans le bain d'incubation. Enfin, il est souhaitable en fin d'expérience de vérifier en histologie l'intégrité de l'endothélium, car une altération de cette structure modifie profondément le transport protéique (voir plus loin).

\section{Transport pariétal de l'albumine}

Concentrations pariétales d'albu- mine. L $\mathrm{L}_{\mathrm{S}}$ concentrations relatives d'albumine dans l'intima sont de l'ordre de $25 \%$ de la concentration plasmatique chez l'homme [18]. Ces concentrations ne sont pas mesurables chez les petits animaux de laboratoire. Lorsque l'endothélium est maintenu intact, les concentrations relatives moyennes dans la média sont inférieures à $1 \%$ dans l'aorte thoracique, chez le lapin [14] et le porc [9]. Ces concentrations semblent plus élevées chez le rat $(2 \%)$ [20] et dans l'artère pulmonaire du lapin (5\%) [21]. Chez l'homme, ces concentrations dans la média varieraient entre l et $10 \%$ [22].

Les techniques de coupes sériées en congélation permettent de mesurer la répartition spatiale de l'albumine marquée dans la média, depuis sa partie juxta-intimale jusqu'à sa partie juxta-adventicielle. On obtient ainsi in vivo une courbe en $\mathrm{U}$ dont les gradients de concentrations interne (côté luminal) et externe (côté adventiciel) peuvent varier en fonction du territoire vasculaire.

Mécanismes du transport. Les faibles valeurs de concentrations relatives d'albumine dans la paroi artérielle s'expliquent de plusieurs façons. D'une part, l'albumine ne pouvant pénétrer dans les cellules, ne peut que se distribuer dans l'espace extracellulaire (environ $40 \%$ du volume de la média). En fait, son volume de distribution ne représente que 10 à $20 \%$ du volume total de la média, inférieur donc à l'espace extracellulaire [19]. Cette différence s'explique par des phénomènes d'exclusion stérique ou électrostatique (répulsion par les glycosaminoglycanes chargés négativement). D'autre part, l'endothélium vasculaire agit comme une puissante barrière, s'opposant au passage des protéines plasmatiques dans la paroi. En effet, après destruction intentionnelle de l'endothélium, aussi bien in vitro qu'in vivo, les concentrations d'albumine dans la paroi augmentent d'un facteur 10 à $15[19,23]$ (figure 2). Dans les conditions de pression élevée, cette augmentation du transport de l'albumine ne s'explique pas seulement par la levée de la barrière endothéliale. L'apparition d'un œedème de la média, liée à une majoration du flux d'eau transpariétal observée 


\section{RÉFÉRENCES}

18. Smith EB, Staples EM. Plasma protein concentrations in interstitial fluid from human aorta. Proc $R$ Soc Lond (Biol) 1982 . $217: 59-75$

19. Tedgui A, Lever MJ. Effect of pressure and intimal damage on ${ }^{131}$-albumin and ${ }^{14} \mathrm{C}$ sucrose spaces in aorta. Am J Physiol 1987 ; 253 : H1530-9.

20. Belmin J, Tedgui A, Michel JB, Juan L. Influence of renovascular hypertension on the in vivo albumin transport in the rat aorta. Clin Exp Hypertens 1988 (à paraître)

21. Caro CG, Jay M, Lever M J. Labelled albumin uptake by rabbit aorta, pulmonary artery and common carotid artery. J Physiol (Lond) $1985 ; 371: 85 \mathrm{P}$

22. Scott PJ, Hurley PJ. The distribution of radioiodinated serum albumin and low density lipoprotein in tissues and the arterial wall. Atherosclerosis $1970 ; 11$ : 77-103.

23. Ramirez CA, Colton CK, Smith KA, Stemerman MB, Lees RS. Transport of 125$]$-albumin across normal and deendothelialized rabbit thoracic aorta in vivo. Arteriosclerosis $1984 ; 4$ : 283-9l.

24. Tedgui A, Lever MJ. Filtration through damaged and undamaged rabbit thoracic aorta. Am J Physiol 1984; H784-91.

25. Duncan LE Jr, Cornfield J, Buck K. The effect of blood pressure on the passage of labelled plasma albumin into canine aortic wall in vivo. I Clin Invest 1962; 41 : 1537-45. 26. Owens GK, Reidy MA. Hyperplasic growth response of vascular smooth muscle cells following induction of acute hypertension in rats by aortic coarctation. Circ Res $1985 ; 57: 695-705$.

27. Owens GK, Schwartz SM Vascular smooth muscle cell hypertrophy and hyperploidy in the Goldblatt hypertensive rat. Circ Res 1983 ; 53 : 491-501.

28. Chobanian AV, Menzoian JO, Shipman J, Health K, Haudenschild CC. Effects of endothelial denudation and cholesterol feeding on in vivo transport of albumin glucose and water across rabbit carotid artery. Circ Res 1983 ; 53 : 805-14.

29. Stefanovitch V, Gore I. Cholesterol diet and permeability of rabbit aorta. Exp $\mathrm{Mol}$ Pathol 1971; 14 : 20-9.

30. Silkworth JB, McLean B, Stehbens WE. The effect of hypercholesterolemia on aortic endothelium studied en-face. Atherosclerosis $1975 ; 22: 335-48$.

31. Stemerman MB. Effects of moderate hypercholesterolemia on rabbit endothelium. Arteriosclerosis 1981 ; 1 : 25-32.

32. Feig LA, Peppas NA, ColtonCK, Smith KA, Lees RS. The effect of angiotensin II on in vivo albumin transport in normal rabbit aortic tissue. Atherosclerosis 1982; 44 : 307-18.

33. Caro CG, Lever MJ. Effect of vasoactive agents and applied stress on the albumin space of excised common carotid arteries. Atherosclerosis $1983 ; 46$ : 137-46.

34. Curmi PA, Tedgui A. Influence of LDL receptor on the uptake and degradation of low density lipoprotein (LDL) in the media of rabbit thoracic aorta. J Physiol (Lond) $1987 ; 388: 22 \mathrm{P}$

35. Fry DL. Mass transport, atherogenesis dans ces conditions [24], entraîne une augmentation du volume de distribution de l'albumine [19].

La forme des profils de concentrations dans la média peut s'expliquer par les mécanismes de transport transpariétal. Le transport des macromolécules à travers l'endothélium se fait via les vésicules de pinocytose. Le transport à travers la média a lieu par diffusion et convection (figure 3). La diffusion des macromolécules est un phénomène de transport bidirectionnel qui s'effectue d'une part à partir de la lumière et d'autre part à partir des vasa vasorum adventiciels vers le centre de la média. Ce transport dépend des gradients de concentrations et des résistances endothéliales (dans l'artère et dans les vasa vasorum), mais aussi de la perméabilité propre de la média. Le transport des macromolécules par convection se fait par entraînement dans le courant d'eau transpariétal, qui s'effectue de la lumière vers l'adventice. Ce phénomène permet d'expliquer la forme des profils transmuraux des concen- trations d'albumine. Le flux d'eau transpariétal influe non seulement sur le mouvement des espèces transportées, mais également sur la configuration de la paroi artérielle [19]. Les rôles respectifs de ces deux phénomènes de transport ont été précisés grâce à des études réalisées in vitro montrant que la convection est aussi, voire plus importante quantitativement que la diffusion [16].

Effet de la pression artérielle. L'effet de la pression artérielle sur le transport d'albumine a pu être étudié sur des artères excisées in vitro. L'augmentation du flux d'eau transpariétal qu'induit l'élévation de pression, accroît la part convective du transport. De plus, l'augmentation du flux d'eau transpariétal peut créer, dans les conditions de dénudation endothéliale, un oedème de la paroi et contribuer ainsi à augmenter les concentrations d'albumine (exprimées par rapport au volume pariétal total) [19]. L'hypertension artérielle aiguë induite chez le chien par section du nerf vague produit une

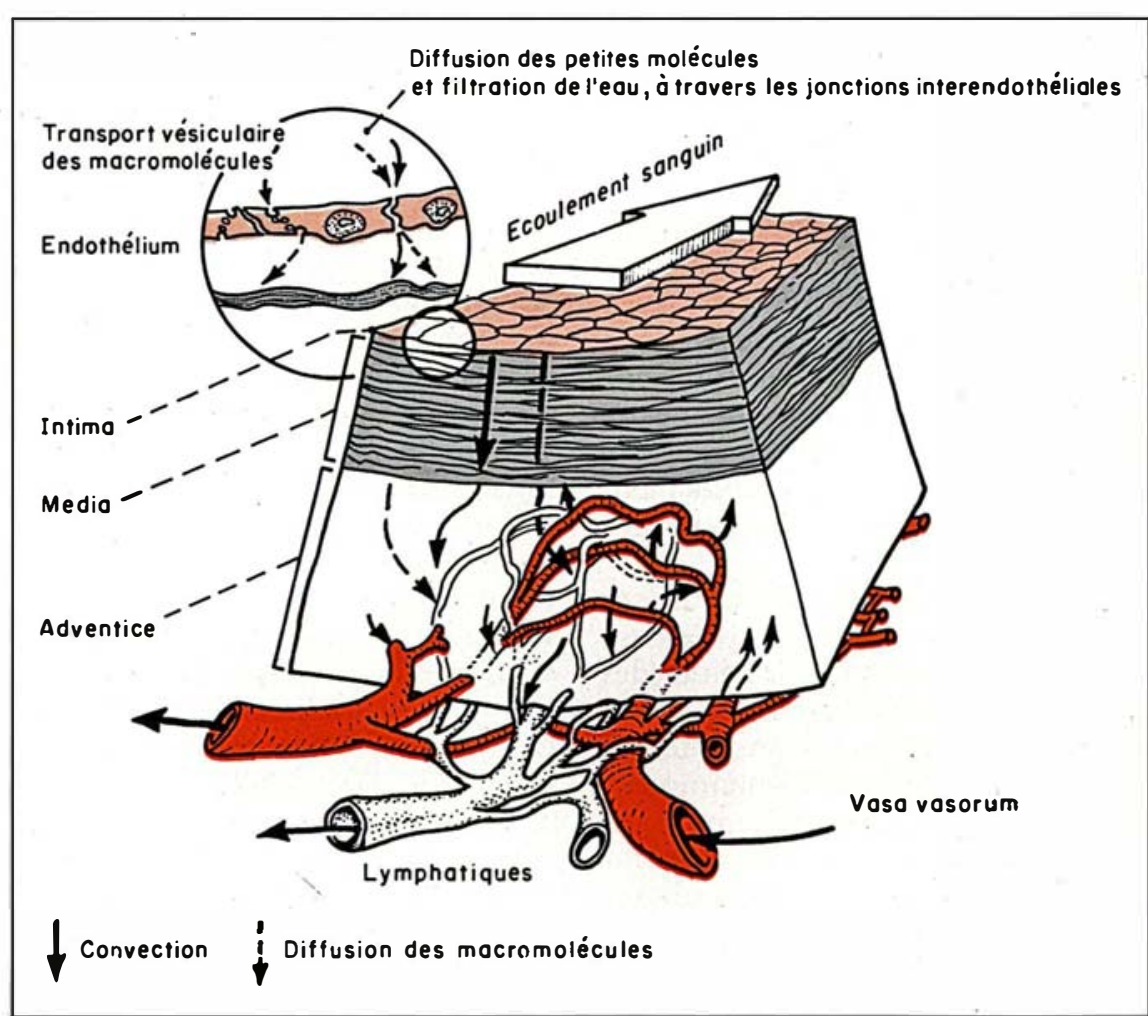

Figure 3. Schéma de la paroi artérielle illustrant les différents modes de transport des macromolécules. 


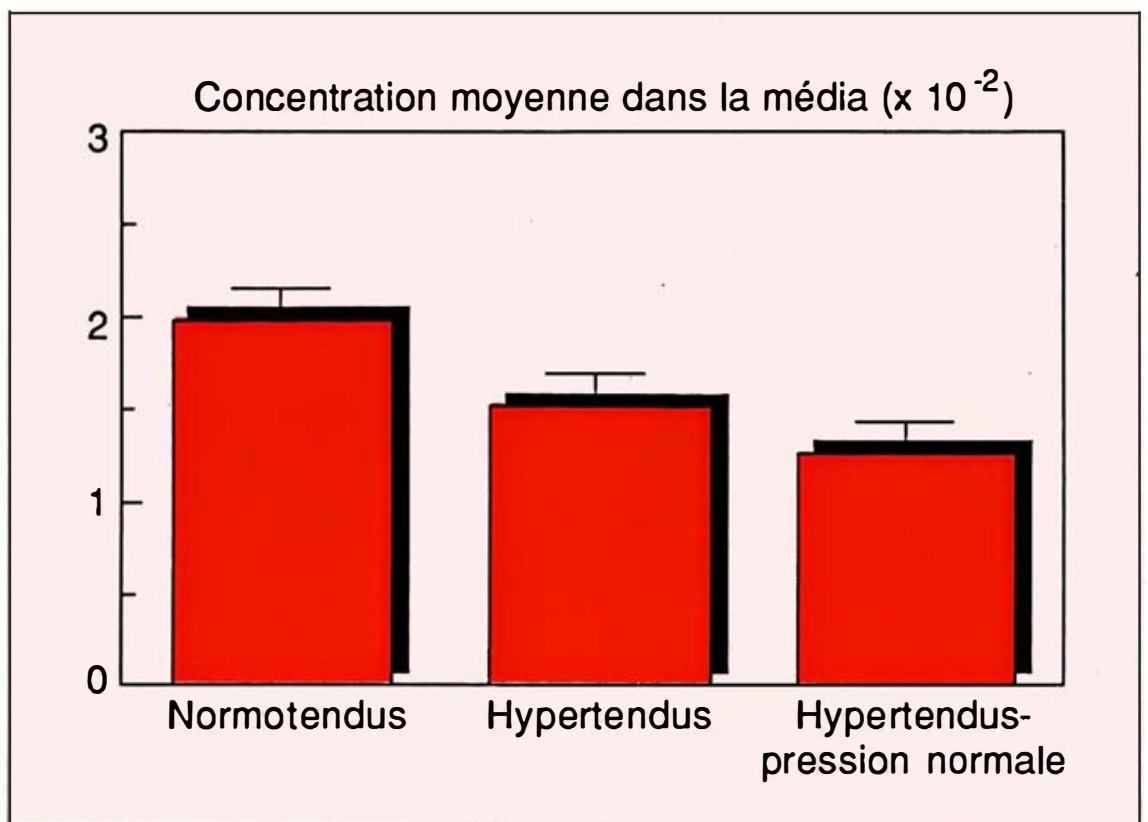

Figure 4. Concentration relative moyenne d'albumine dans la média de l'aorte de rats normotendus, hypertendus et hypertendus dont la pression artérielle a été normalisée pendant la durée de la mesure de la captation d'albumine.
La surcharge en cholestérol crée des modifications structurales de l'endothélium qui pourraient expliquer une augmentation de sa perméabilité [30], mais des altérations fonctionnelles de l'endothélium (pinocytose accrue) pourraient aussi être en cause [31].

Certains agents vaso-actifs modifient de façon importante le transport macromoléculaire. L'angiotensine II augmente la perméabilité endothéliale et augmente le transport d'albumine dans la crosse aortique chez le lapin, mais sans le modifier dans l'aorte thoracique descendante [32]. La noradrénaline [33] et la contraction induite par le $\mathrm{KCl}$ [17] augmentent la perméabilité de l'endothélium mais diminuent celle de la média. Les dérivés nitrés [33] et les inhibiteurs calciques [17] ont tendance à augmenter la perméabilité de la média.

\section{Transport pariétal des $L D L$}

De nombreuses études effectuées in vitro [34, 35] ou in vivo [14, 36] et dans différentes espèces (lapin, rat, chien, porc) ont montré que les LDL pénètrent dans la paroi artérielle. Dans l'aorte humaine, Scott et Hurley[22] ont injecté des LDL marquées à des patients en coma dépassé. Les concentrations les plus élevées s'observaient dans la crosse et dans l'aorte abdominale et augmentaient avec la durée de l'étude (16 jours au maximum). Dans l'aorte de lapin, les LDL pénètrent très rapidement à partir des faces luminale et adventicielle et leur concentration devient stationnaire après quatre heures [15]. Les profits transmuraux des LDL d'aspect superposable à ceux de l'albumine [14, 15] témoignent d'un mécanisme de transport semblable pour les deux espèces.

Concentration intimale en LDL. La concentration en LDL dans l'intima d'aortes humaines est environ le double de la concentration plasmatique, tandis que la concentration d'albumine n'est que le quart [5]. Dans la média interne, il n'existe que des traces de LDL, alors que la concentration d'albumine est de l'ordre de $7 \%$ de la concentration plasmatique. La conclusion qu'en tirent Smith et Staples [5] est que, dans l'aorte 
humaine, la concentration en protéines plasmatiques est proportionnelle au poids moléculaire dans l'intima, et inversement proportionnelle dans la média. Après incubation, dans du sérum autologue, d'intima d'aortes sur lesquelles la limitante élastique interne avait été détruite, la concentration intimale en LDL avait diminué et la concentration en albumine augmenté [18]. Il apparaît donc que la majeure partie des LDL se trouvent dans l'intima sous une forme diffusible et qu'elles sont piégées par la barrière sélective que constitue la limitante élastique interne et non par liaison spécifique. La diffusion simple ne peut néanmoins expliquer l'existence d'un gradient de concentration positif entre la source (le plasma) et le puits (l'intima), malgré la présence d'une barrière de diffusion (la limitante élastique interne). Ce gradient s'explique par l'importance d'un flux convectif qui s'oppose à la rétrodiffusion des LDL vers le plasma, lorsque la concentration intimale dépasse celle du plasma [35].

Rôle du récepteur des LDL. Pour étudier le rôle des récepteurs membranaires des LDL, dans le transport pariétal des LDL, des expériences ont été réalisées en utilisant des LDL natives et des LDL méthylées qui ne sont pas reconnues par le récepteur spécifique des LDL, ce qui a permis de mettre en évidence le rôle important de ce récepteur dans la captation et la dégradation de ces lipoprotéines par la média aortique du lapin [34].

\section{Transport protéique et athérogenèse}

Compte tenu des modalités du transport macromoléculaire à travers la paroi artérielle, on peut retenir plusieurs facteurs susceptibles d'augmenter les concentrations de LDL dans l'intima: (a) une augmentation de leur concentration plasmatique; (b) une augmentation de la perméabilité endothéliale; (c) une augmentation de la pression artérielle (augmentant le flux convectif) ; (d) une diminution de leur élimination dans la paroi, cette élimination pouvant se faire, soit par une dégradation intracellulaire liée à la pré- aux LDL, soit par drainage à travers la média vers l'adventice et l'espace extravasculaire.

On peut ainsi comprendre comment certains facteurs de risque des maladies liées à l'athérome sont susceptibles d'entraîner une accumulation de LDL dans l'intima. Dans l'hypercholestérolémie, les concentrations plasmatiques en LDL et la perméabilité de l'endothélium sont accrues. De plus, la dégradation des LDL par les cellules de la paroi peut être réduite du fait d'une diminution du nombre des récepteurs membranaires aux LDL (soit d'origine génétique, soit par down regulation). L'hypertension artérielle est responsable d'une augmentation du transport convectif et d'une baisse importante de la perméabilité de la média aux macromolécules, ce qui peut réduire leurs possibilités de drainage à travers cette structure et favoriser leur accumulation dans l'espace sousendothélial. De plus, certains agents vaso-actifs tels que l'angiotensine II ou les catécholamines mis en jeu dans certaines formes d'hypertension pourraient encore accentuer ce phénomène, en augmentant la perméabilité endothéliale tout en diminuant celle de la média. On peut noter que l'hypercholestérolémie et l'hypertension artérielle modifient le transport macromoléculaire selon des mécanismes différents, ce qui pourrait expliquer en partie le caractère additif de ces facteurs de risque.

Les effets sur le transport protéique d'autres facteurs de risque tels que le diabète, le tabagisme ou le vieillissement de la paroi artérielle nécessiteraient d'être étudiés. De même, une étude des différences de transport entre territoires vasculaires et de leur origine permettrait peut-être de mieux comprendre la prédilection de l'athérome pour certains sites.

Enfin, un déséquilibre du transport macromoléculaire et l'accumulation intimale de LDL ne représentent probablement qu'un aspect de l'athérogenèse dont le caractère multifactoriel n'est plus discuté. Cette conception du transport des LDL et de l'infiltration lipidique n'exclut aucunement les autres conceptions pathogéniques qui sont au contraire complémentaires, permettant d'expliquer l'évolution de la strie lipidique vers la plaque d'athérome

\section{Summary}

Damage to the endothelium and increased influx of lipoproteins have long been considered as the first events in atherogenesis. It has been, however, demonstrated that atheromatous plaque develops in the absence of endothelial denudation. Moreover, atherosclerosis is an arterial disease which occurs in relatively thick-walled arteries and not in veins. This suggests the possible importance of transport phenomena within the wall. Intimal accumulation of plasma constituents may result from an imbalance between influx and efflux. The influx is controlled by the transport across the endothelium and the efflux by transport across the medial layer, the media offering a barrier to the drainage of macromolecules from the subendothelial space to the adventitial vasa vasorum and lymphatics. The resistance to the transport opposed by the media is influenced by various factors, including arterial blood pressure, pressure-induced structural changes of the wall and vasoactive agents. It is the subject of this brief review to report investigations in the field of the biology of the arterial wall concerned with the transport of macromolecules.

\section{TIRÉS A PART}

A. Tedgui. 